\title{
Baroreflex Sensitivity Evolution Before Weaning From Mechanical Ventilation
}

\author{
Pablo Armañac ${ }^{1,2}$, David Hernando ${ }^{1,2}$, Jesús Lázaro ${ }^{1,2}$, Candelaria de Haro ${ }^{3,4}$, \\ Rudys Magrans ${ }^{5}$, Leonardo Sarlabous ${ }^{3}$, Josefina López-Aguilar ${ }^{3,4}$, \\ Pablo Laguna ${ }^{1,2}$, Eduardo Gil ${ }^{1,2}$, Lluís Blanch ${ }^{3,4}$, Raquel Bailón ${ }^{1,2}$ \\ ${ }^{1}$ BSICoS group, Instituto de Investigacion en Ingeniería de Aragón, Zaragoza, Spain \\ ${ }^{2}$ CIBER de Bioingeniería, Biomateriales y Nanomedicina (CIBER-BBN), Madrid, Spain \\ ${ }^{3}$ Critical Care Center, Hospital i Institut d'Investigació Parc Taulí I3PT, Sabadell, Spain \\ ${ }^{4}$ CIBER Enfermedades Respiratorias (CIBER-ES), Instituto de Salud Carlos III, Madrid, Spain \\ ${ }^{5}$ BetterCare, Barcelona, Spain
}

\begin{abstract}
Weaning is the process of withdrawing mechanical ventilation at the Intensive Care Units. The problem is that around $20 \%$ of weaned patients were not actually ready for discontinuation. Studies suggest that vagal dysfunction is lower in patients successfully weaned. Therefore, the Baroreflex Sensitivity (BRS) and Heart Rate Variability $(H R V)$ are estimated to see if they can provide additional information to improve the prediction of weaning outcomes.

9 successfully weaned patients (S-group) and 6 unsuccessfully weaned (F-group) were monitored in the last hour prior to the Spontaneous Breathing Trial. The BRS is estimated through spectral analysis, to obtain the $\alpha$ parameter in the low and high frequency bands, and through the capacity, $C$, estimated by the Bivariate Phase Rectified Signal Average (BPRSA) method. The current clinic parameters of weaning readiness do not show statistical differences. However, the capacity to changes of the BRS, $C$, estimated via BPRSA, exhibits significant differences between the two groups. Negative values of $C$, and with higher absolute values, were obtained for the $S$-group. Temporal indices of HRV also show differences, but not significant. These results suggest that BRS should be further explored for predicting weaning outcomes.
\end{abstract}

\section{Introduction}

Patients admitted to Intensive Care Units (ICU) generally need the support of Mechanical Ventilation (MV) to maintain breathing. Weaning is the process of withdrawing this MV. It is a challenging and very delicate procedure and it must be considered as soon as possible [1]. However, weaning failure and reintubation after a premature weaning is really critical and can lead to an increased risk of severe respiratory complications such as pneumonias, lung injuries, or diaphragmatic dysfunctions. Indeed, weaning failure increases the risk of mortality in the range from 25\% to 50\%. The Spontaneous Breathing Trial (SBT) is the diagnostic test to determine weaning readiness [1], and some parameters based on respiration, cardiovascular status and hemodynamics help clinicians to determine if a weaning attempt will be successful or not.

The cardiovascular control involves several mechanisms to dynamically couple arterial pressure, heart rate and respiration. The cross-talk between heart, lungs and nervous system is mainly coordinated by the Autonomic Nervous System (ANS). Indices derived from Baroreflex Sensitivity (BRS) and Heart Rate Variability (HRV) have already been proven to assess the regulatory mechanisms of the ANS [2]. In fact, recent studies revealed high incidence of autonomic dysfunction in patients admitted to the ICU, resulting in chronic adrenergic activations and acute respiratory distress syndromes [3]. However, no previous work investigated the role of the BRS. Moreover, none looked for differences just before the SBT, in order to improve the predictive value of the weaning indices.

This study aims to evaluate the BRS in the last hour prior to the SBT and explore if this can provide additional information to improve the prediction of weaning outcomes. Different approaches exist to assess BRS. In this work, the BRS will be calculated via the spectral analysis of HRV and SAP, to derive the well-known $\alpha$ index [2]. Furthermore, the method referred to as Bivariate Phase Rectified Signal Averaging (BPRSA) will also be computed [4].

\section{Materials and Methods}

\subsection{Data set}

Patients were monitored in the ICU's attached to the Hospital Universitari Parc Tauli of Sabadell (Barcelona), during the hour prior to the SBT. Patients with neurological disorder, dementia or focal brain injury at ICU admission 
were excluded for the analysis. For this study, only those patients ventilated with assist/support ventilation modes during the last hour were considered.

When the clinical status and health condition of a patient improved in the ICU, they are considered presumably ready for weaning [1]. These patients had to perform the SBT. The SBT was carried out by a T-tube test or by low-level inspiratory pressure support. The criteria in [1], shows the indicators used to evaluate the success of the SBT, in order to decide if MV could be withdrawn. Weaning was considered successful for patients who passed SBT [1], and did not required reintubation or reconnection to MV in the 48 hours after SBT. These patients are in the successful weaning group, namely $S$-group. Patients presenting at least one item of the intolerance criteria [1], were not ready for discontinuation and weaning failure was considered. These patients were classified in the F-group. With all this premises, 9 patients from the $S$-group and 6 from the $F$-group remained.

Physiological signals were recorded using the proprietary system BetterCare ${ }^{\circledR}$. This system for data collection standardizes and synchronizes all the biosignals from all the different bedside monitors and ventilators. The signals are sampled at 200 samples per second. The present study uses the recordings of the lead II of the electrocardiogram (ECG), the blood pressure (BP) signal, acquired using an invasive arterial catheter, and the respiratory frequency signal, $f_{r}(t)$.

\subsection{HRV estimation}

The ECG signal is upsampled at $1000 \mathrm{~Hz}$ with cubic spline interpolation. Then, the R-waves are detected by means of a wavelet-based method [6]. Detections are manually corrected using the R-DECO application [7]. Once ECG detections are correct, the RR interval is defined by the time between two consecutive R-waves, and the normal-to-normal (NN) interval is defined after correcting ectopic beats [8]. From the NN series, temporal indices of HRV are calculated: standard deviation of the NN intervals (SDNN) and the root mean-square of successive differences (RMSSD).

In order to explain the regulation of the heart rate by the ANS, the HRV signal is estimated using the Time-Varying Integral Pulse Frequency Modulation model [8]. Given a particular beat time occurrence series, the instantaneous $\mathrm{HR}, d_{\mathrm{HR}}(t)$, can be derived and expressed as $d_{\mathrm{HR}}(t)=$ $(1+m(t)) / T(t)$. The term $m(t)$ represents the modulating signal, which is assumed to contain the ANS modulation. The instantaneous mean $\mathrm{HR}, d_{\mathrm{HRM}}(t)=1 / T(t)$, is obtained by low-pass filtering $d_{\mathrm{HR}}(t)$ at $0.03 \mathrm{~Hz}$. The HRV signal is represented by the term $d_{\mathrm{HRV}}(t)=d_{\mathrm{HR}}(t)-$ $d_{\text {HRM }}(t)$. Finally, the modulating signal is estimated as $m(t)=d_{\mathrm{HRV}}(t) / d_{\mathrm{HRM}}(t)$. The evenly-sampled version, $m(n)$, is obtained by resampling $m(t)$ at $f_{s}=4 \mathrm{~Hz}$.

Since respiration affects HRV and SAP through changes in the respiratory frequency and the respiratory pattern, the analysis is performed guided by respiration. Therefore, the High Frequency (HF) band is redefined to be centred at the respiratory frequency, $f_{r}$, and time-varying: $\Omega_{\mathrm{HF}}(t)=$ $\left[f_{r}(t)-0.15, f_{r}(t)+0.15\right] \mathrm{Hz}$. This is mainly motivated since $f_{r}$ in MV patients, is around 24 respirations per minute (rpm), and this is in the limit of the classic HF band. The HF power, $\mathrm{P}_{\mathrm{HF}}$, is defined as the power within the HF band. Low Frequency (LF) power, $\mathrm{P}_{\mathrm{LF}}$, is defined as the power in the classic LF band: $\Omega_{\mathrm{LF}}=[0.04,0.15]$ Hz. The sympathovagal balance is estimated with the normalized power: $\mathrm{P}_{\mathrm{LF}}^{n}=\mathrm{P}_{\mathrm{LF}} /\left(\mathrm{P}_{\mathrm{LF}}+\mathrm{P}_{\mathrm{HF}}\right)$.

\subsection{SAP estimation and BRS analysis}

An ad hoc algorithm is implemented to delineate the BP signal. The SAP values are essentially detected using a pulse detection algorithm based on a low pass differentiator (LPD) filter [9], and a time-varying threshold defined by an enveloping procedure [7].

First, the BP signal, $x_{\mathrm{BP}}(n)$, is filtered using the LPD filter, to accentuate the maximum up-slope of the pulses. This signal is referred to as $x_{\mathrm{BP}}^{\prime}(n)$. After that, the enveloping procedure computes the upper and lower envelope of the filtered BP signal, $x_{\mathrm{BP}}^{\prime}(n)$. A flattened-positive version, $x_{\mathrm{F}}(n)$, is obtained, which enhances the up-slopes. The location of the pulses, $n_{\mathrm{D}_{i}}$, are found by detecting the peaks in this flattened version, applying the adaptive thresholding procedure of the Pan-Tompkins algorithm [7]. Then, once the locations of the pulses, $n_{\mathrm{D}_{i}}$, are identified, the BP signal is used to obtain, at the maximum of each pulse, $n_{\mathrm{SAP}_{i}}$, the corresponding SAP value, $x_{\mathrm{BP}}\left(n_{\mathrm{SAP}_{i}}\right)$. Erroneous SAP detections are manually corrected using the R-DECO as well [7]. An illustration of the procedure is in Fig. 1.

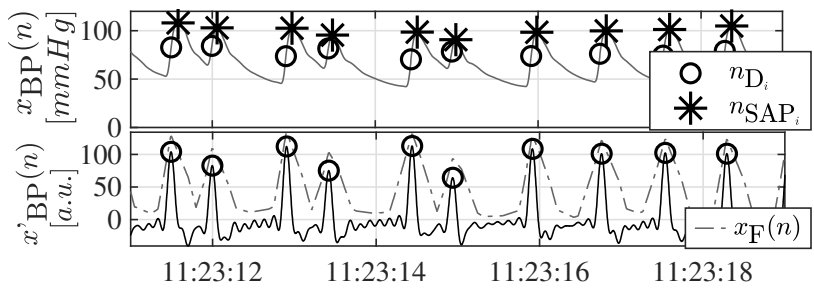

Figure 1. Blood Presure Delineation. The LPD-filtered $\mathrm{BP}$ signal, $x_{\mathrm{BP}}^{\prime}(n)$, is in bottom. The flattened-positive envelope, $x_{\mathrm{F}}(n)$, of $x_{\mathrm{BP}}^{\prime}(n)$ is represented with the dasheddotted line. From $x_{\mathrm{F}}(n)$, the location of the pulses, $n_{\mathrm{D}_{i}}$, are identified. The BP signal, $x_{\mathrm{BP}}(n)$, plotted in the top, is used to obtain at the maximum of each pulse, $n_{\mathrm{SAP}_{i}}$, the corresponding SAP value, $x_{\mathrm{BP}}\left(n_{\mathrm{SAP}_{i}}\right)$, for each heartbeat.

Now, the SAP signal, $d_{\text {SAP }}^{u}(n)$, can be estimated as:

$$
d_{\mathrm{SAP}}^{u}(n)=\sum_{i}\left[x_{\mathrm{BP}}\left(n_{\mathrm{SAP}_{i}}\right)\right] \delta\left(n-n_{\mathrm{SAP}_{i}}\right)
$$


where $\delta(\cdot)$ denotes the Kronecker delta function, and the superscript " $u$ " denotes that the signal is unevenly sampled, since heartbeats occur unevenly in time. The evenlysampled version, $d_{\mathrm{SAP}}(n)$, is obtained by cubic spline interpolation at $f_{s}=4 \mathrm{~Hz}$.

At this point, the BRS indices based on the $\alpha$ index can be calculated from the spectral analysis of HRV and SAP:

$$
\alpha_{\mathrm{B}}(n)=\sqrt{\frac{\int_{\Omega_{\mathrm{B}}} \hat{S}_{\mathrm{HRV}}(n, f) d f}{\int_{\Omega_{\mathrm{B}}} \hat{S}_{\mathrm{SAP}}(n, f) d f}}, B \in\{L F, H F\},
$$

where $\hat{S}_{\mathrm{HRV}}(n, f)$ and $\hat{S}_{\mathrm{SAP}}(n, f)$ are the time-varying power spectral densities of $m(n)$ and $d_{\mathrm{SAP}}(n)$, respectively, calculated by means of the Cohen's Class Wigner Ville Distribution [10], with the parameters adjusted for a time and frequency resolution of $11.25 \mathrm{~s}$ and $0.039 \mathrm{~Hz}$, respectively. This time-frequency distribution is used to deal with non-stationarities, since recordings are 1-hour length.

\subsection{BPRSA analysis}

An alternative method to analyse BRS function has been proposed based on BPRSA [4]. Essentially, an averaged HRV profile is obtained of the overall heart response to SAP increases. For this, anchor points (AP) must be identified firstly in the $d_{\text {SAP }}(n)$ series. Usually, AP's are defined in samples where the average of their $T$ prior samples is greater than the average of their $T$ subsequent samples [4]:

$$
\frac{1}{T} \sum_{i=0}^{T-1} d_{\mathrm{SAP}}(n+i)>\frac{1}{T} \sum_{i=1}^{T} d_{\mathrm{SAP}}(n-i)
$$

However, in this work, AP's are considered only if they are also local maxima in the corresponding series of Eq. (3). With this approach, only one AP is defined for each upslope. Now, windows of length $2 L$ are segmented around each AP over the HRV series. Finally, the BPRSA curve is obtained by averaging all segmented windows in the HRV. In this study, $L$ is set to $10^{*} f_{s}$. $T$ sets an upper frequency limit for the periodicities that can be detected by BPRSA [4]. For each patient, $T$ is adjusted with their mean respiratory frequency, $T \approx f_{s} /\left(2.5 \cdot \overline{f_{r}}\right)$. An illustrative example of the methodology is shown in Fig. 2.

The BRS, estimated from the BPRSA curve, is quantified with the capacity term, $C$ [11]:

$$
C=\frac{1}{2 s} \sum_{i=1}^{s} \operatorname{BPRSA}(L+i)-\frac{1}{2 s} \sum_{i=0}^{s-1} \operatorname{BPRSA}(L-i) \text {. }
$$

The index $C$ is based on the Haar wavelet, but evaluated at a scale $s$ and location $(L+1)$, in the center of the curve. Then, $C$ can be either positive or negative. The scale, $s$, selects the oscillations in the BPRSA curve that most affect $C$. In this study, $s$ is taken to be equal to $T$, since using $s=T$ avoids the need of optimization [11].

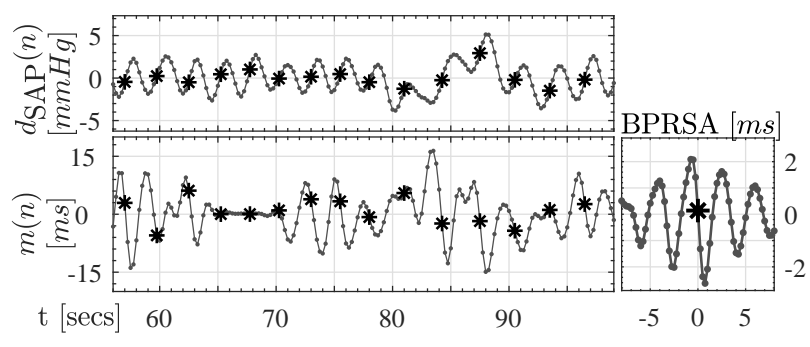

Figure 2. Estimation of the BPRSA curve for a patient. In top-left, the SAP signal, $d_{\mathrm{SAP}}(n)$, as driver mechanism, in which AP's (asterisks) are defined. In bottom-left, HRV signal, $m(n)$. Around each AP, segments of $2 L$ length are extracted from the HRV signal. In bottom-right, the BPRSA curve, obtained averaging all segments of HRV.

\subsection{Statistical analysis}

The parameters $\overline{\mathrm{HR}}, \overline{\mathrm{SAP}}, \overline{f_{r}}, \mathrm{SDNN}, \mathrm{RMSSD}, \overline{\alpha_{\mathrm{LF}}}, \overline{\alpha_{\mathrm{HF}}}$, $\overline{\mathrm{P}_{\mathrm{LF}}^{n}}$ and $\bar{C}$ are averaged for the whole recordings of 1 -hour (Tab. 1). In addition, $\alpha_{\mathrm{LF}}, \alpha_{\mathrm{HF}}, \mathrm{P}_{\mathrm{LF}}^{n}$ and $C$, are also averaged in periods of 5 minutes (Fig. 3). The Mann-Whitney U-test is used to compare the values of the $S$-group vs. $F$ group. Statistical significance is considered for $p \leq 0.05$.

\section{Results and Discussion}

The results for the scalar variables are in Tab. 1. The capacity, $\bar{C}$, obtained from the BPRSA curve, is the only parameter significantly different comparing $S$-group vs $F$ group. No significant differences can be found for SDNN and RMSSD of the HRV, but they are notably higher for the $S$-group. In fact, other works did found significant differences for these between the two groups when comparing values half an hour before the SBT, and half an hour after SBT [3].

Table 1. Scalar indices. Inter-subject median $\left(Q_{1}, Q_{3}\right)$, computed throughout the whole hour before SBT.

\begin{tabular}{c|cc|l}
\hline \hline & S-group & F-group & $p$ \\
\hline$\overline{\mathrm{HR}}[\mathrm{bpm}]$ & $80(75,89)$ & $84(81,123)$ & 0.33 \\
$\overline{\mathrm{SAP}}[\mathrm{mmH}]$ & $132(110,139)$ & $126(101,141)$ & 0.78 \\
$\overline{f_{r}}[\mathrm{rpm}]$ & $19(18,23)$ & $22(18,24)$ & 0.61 \\
$\mathrm{SDNN}[\mathrm{ms}]$ & $32(27,53)$ & $22(15,26)$ & 0.11 \\
$\mathrm{RMSSD}[\mathrm{ms}]$ & $11(6,14)$ & $5(3,8)$ & 0.18 \\
$\overline{\alpha_{\mathrm{LF}}}[\mathrm{ms} / \mathrm{mmHg}]$ & $6.0(3.5,11.8)$ & $5.1(2.3,7.1)$ & 0.46 \\
$\overline{\alpha_{\mathrm{HF}}}[\mathrm{ms} / \mathrm{mmHg}]$ & $7.1(3.1,8.7)$ & $6.7(1.6,8.6)$ & 0.78 \\
$\overline{\mathrm{P}_{\mathrm{LF}}^{n}}$ & $0.56(0.30,0.65)$ & $0.66(0.41,0.80)$ & 0.39 \\
$\bar{C}[\mathrm{~ms}]$ & $-1.7(-2.7,-0.8)$ & $0.9(0.5,1.4)$ & $\mathbf{0 . 0 2}$ \\
\hline \hline
\end{tabular}

Since no strong conclusions can be extracted from the averages of the whole hour (see Tab. 1), the evolution of $\alpha_{\mathrm{LF}}, \alpha_{\mathrm{HF}}, \mathrm{P}_{\mathrm{LF}}^{n}$ and $C$ is calculated in 5-minutes averagedperiods, throughout the hour before SBT (Fig. 3). Clear statistical significant differences in $C$ are visible comparing the $S$-group vs $F$-group in many 5 -minutes periods. 


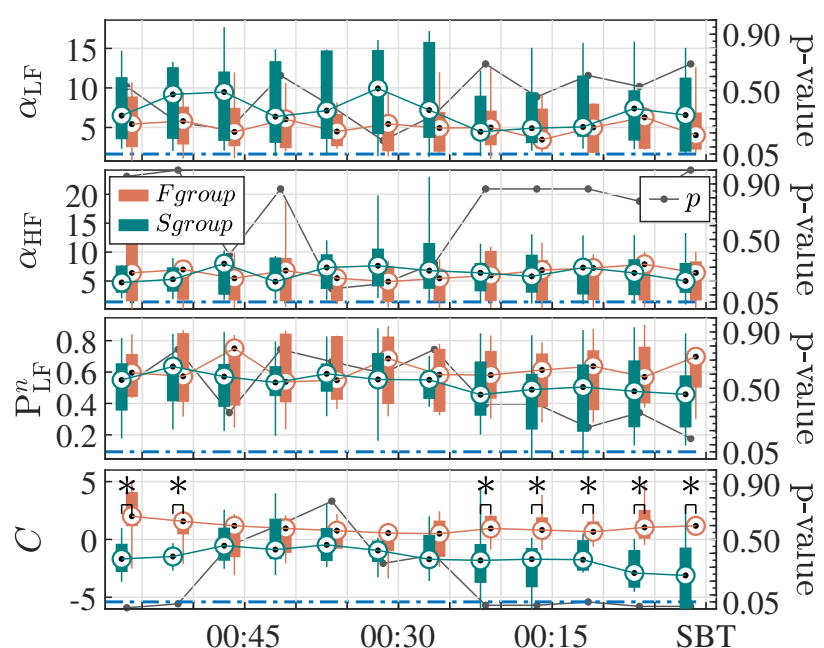

Figure 3. Evolution, throughout the hour before SBT, of the BRS with $\alpha_{\mathrm{LF}}$ and $\alpha_{\mathrm{HF}}$, in $[m s / m m H g], \mathrm{P}_{\mathrm{LF}}^{n}$ and $C$ in $[m s]$. Green and Red boxplots represent the average of each 5-minutes period for the $S$-group and $F$-group, respectively. The $p$-value is represented in the right axis, from 0 to 1 , comparing each 5-minutes period and asterisk represents statistical significance.

Again, no significant differences are found in any period for $\alpha_{\mathrm{LF}}, \alpha_{\mathrm{HF}}$ or $\mathrm{P}_{\mathrm{LF}}^{n}$ either. In spite of that, the $\alpha_{\mathrm{LF}}$ median values are generally higher for the $S$-group during the whole hour.

Maybe, no strong differences can be found since these patients present strong non-linear dynamics because of the disease severity [5]. This fact reflects complex influences of respiration on HR and SAP variability and might limit the ability of $\alpha_{\mathrm{LF}}$ and $\alpha_{\mathrm{HF}}$ for BRS assessment, even though analysis was guided by respiration.

It is important to remark that $C$ in absolute values, is higher for the $S$-group. This could be because of the effect of the strength of SAP changes in HRV, reflected in the amplitude of the BPRSA curve. Besides, notice that $C$ is negative for the $S$-group but positive for the $F$-group, since sympathetic activity is associated with higher latency shifts in the response of the HRV for the spontaneous increases in BP. In fact, related to this, higher adaptability to changes can be deduced from SDNN and higher parasympathetic activity from RMSSD, for the $S$-group. Note that the scale $s=T$, used for the computation of $C$, is not distorting the results, since $\overline{f_{r}}$ is similar for both groups (see Tab. 1).

The selection of the AP's for the BPRSA analysis is of relative importance. Using all the increasing samples as AP's $[4,5]$, produces a reduction in the amplitude of the average curve, because of the distortion introduced by the average of few-samples-delayed ensembles. This fact compromises the BPRSA curve, and some investigation should be done in order to standardize the selection of AP's.

\section{Conclusions}

BRS was evaluated in a sample of critical patients in MV. Results revealed that the capacity of the BRS, measured via BPRSA analysis, is stronger and with negative values for patients who were actually ready for weaning.

Results suggest that BRS quantification is potentially useful for predicting weaning outcomes in the ICU. Future studies will provide useful insights of the role of the ANS in the weaning process that will help reduce weaning failure rates and the very adverse effects of reintubation.

\section{Acknowledgements}

This work was supported by CIBER-BBN and CIBERES through Instituto de Salud Carlos III; by MINECO (RTI2018-097723-B-I00); by Gobierno de Aragón (Reference Group BSICoS T39-20R) cofunded by FEDER 2014-2020; an also from EU H2020 under the Marie Skłodowska-Curie Grant No. 745755.

\section{References}

[1] Boles JM, et al. Weaning from mechanical ventilation. Eur Resp J 2007;29(5).

[2] La Rovere MT, et al. Baroreflex sensitivity: measurement and clinical implications. Ann NInv Electrocard 2008;13(2).

[3] Chen YJ, et al. Vagal withdrawal and psychological distress during ventilator weaning and the related outcomes. J Psychosom R 2017;101.

[4] Müller A, et al. Bivariate phase-rectified signal averaging for assessment of spontaneous baroreflex sensitivity: normalization of the results. J Electrocard 2012;45(1).

[5] Pinna GD, et al. Different estimation methods of spontaneous baroreflex sensitivity have different predictive value in heart failure patients. J Hypertension 2017;35(8).

[6] Martínez JP, et al. A wavelet-based ECG delineator: evaluation on standard databases. IEEE TBME 2004;51(4).

[7] Moeyersons J, et al. R-DECO: An open-source matlab based graphical user interface for the detection and correction of Rpeaks. PeerJ 2019;5.

[8] Mateo J, Laguna P. Analysis of heart rate variability in the presence of ectopic beats using the heart timing signal. IEEE TBME 2003;50(3).

[9] Lázaro J, et al. Pulse rate variability analysis for discrimination of sleep-apnea-related decreases in the amplitude fluctuations of pulse photoplethysmographic signal in children. IEEE JBHI 2014;18(1).

[10] Orini M, et al. Characterization of dynamic interactions between cardiovascular signals by time-frequency coherence. IEEE TBME 2011;59(3).

[11] Sassi R, et al. A methodological assessment of phase-rectified signal averaging through simulated beat-to-beat interval time series. In CinC. 2014;

Address for correspondence:

Pablo Armañac

Dep. Ingeniería Electrónica y Comunicaciones. Universidad de Zaragoza, C/ María de Luna 1, L.3.07, 50018 Zaragoza, Spain parmanac@unizar.es 